\title{
Microbial Community of Tannery Wastewater Involved in Nitrification Revealed by Illumina MiSeq Sequencing ${ }^{\mathbb{S}}$
}

\author{
Xiaojian $\mathrm{Ma}^{1,2}$, Chongde $\mathrm{Wu}^{2 *}$, Huang Jun ${ }^{2}$, Rongqing $\mathrm{Zhou}^{2}$, and $\mathrm{Bi} \mathrm{Shi}^{1 *}$ \\ ${ }^{1}$ National Engineering Laboratory of Clean Technology for Leather Manufacture, Sichuan University, Chengdu 610065, China \\ ${ }^{2}$ College of Light Industry, Textile and Food Engineering, Sichuan University, Chengdu 610065, China
}

\author{
Received: December 19, 2017 \\ Revised: June 2, 2018 \\ Accepted: June 10, 2018 \\ First published online \\ June 8, 2018 \\ ${ }^{*}$ Corresponding authors \\ C.W. \\ Phone: +86-28-85400356 \\ Fax: +86-28-85400356; \\ E-mail: cdwu@scu.edu.cn \\ B.S. \\ Phone: +86-28-85400356; \\ Fax: +86-28-85400356; \\ E-mail: shibi@scu.edu.cn \\ $\mathrm{S}$ upplementary data for this \\ paper are available on-line only at \\ http://jmb.or.kr. \\ pISSN 1017-7825, eISSN 1738-8872 \\ Copyright(C) 2018 by \\ The Korean Society for Microbiology \\ and Biotechnology
}

\begin{abstract}
The aim of this study was to investigate the microbial community of three tannery wastewater treatment plants (WWTPs) involved in nitrification by Illumina MiSeq sequencing. The results showed that highly diverse communities were present in tannery wastewater. A total of six phyla, including Proteobacteria (37-41\%), Bacteroidetes (6.04-16.80), Planctomycetes (3.6516.55), Chloroflexi (2.51-11.48), Actinobacteria (1.91-9.21), and Acidobacteria (3.04-6.20), were identified as the main phyla, and Proteobacteria dominated in all the samples. Within Proteobacteria, Beta-proteobacteria was the most abundant class, with the sequence percentages ranging from $9.66 \%$ to $17.44 \%$. Analysis of the community at the genus level suggested that Thauera, Gp4, Ignavibacterium, Phycisphaera, and Arenimonas were the core genera shared by at least two tannery WWTPs. A detailed analysis of the abundance of ammonia-oxidizing bacteria (AOB) and nitrite-oxidizing bacteria (NOB) indicated that Nitrosospira, Nitrosomonas, and Nitrospira were the main AOB and NOB in tannery wastewater, respectively, which exhibited relatively high abundance in all samples. In addition, real-time quantitative PCR was conducted to validate the results by quantifying the abundance of the $\mathrm{AOB}$ and total bacteria, and similar results were obtained. Overall, the results presented in this study may provide new insights into our understanding of key microorganisms and the entire community of tannery wastewater and contribute to improving the nitrogen removal efficiency.
\end{abstract}

Keywords: Microbial community, nitrification, tannery wastewater, high-throughput sequencing

\section{Introduction}

Tannery wastewater commonly contains a large amount of ammonia nitrogen $\left(\mathrm{NH}_{3}-\mathrm{N}\right)$ and total nitrogen, which mainly originate from unhairing/liming, deliming, and bating processes due to the addition of ammonium salts [1]. Nitrogen removal is one of the most important targets of wastewater treatment. Generally, there are two ways to reduce the nitrogen content from tannery wastewater; namely, elimination at the origins and end-of-pipe treatment [2]. The end-of-pipe treatment, including the processes of nitrification and denitrification, is regarded as an effective strategy to remove nitrogen pollution [3, 4].

Activated sludge is a widely applied biological process in wastewater treatment plants (WWTPs) for treating both municipal and industrial wastewater [4,5]. During the process of nitrogen removal, ammonia is oxidized to nitrite/nitrate via nitrification, and then to nitrogen gas via denitrification by activated sludge. Microorganisms in the active sludge are the main contributors for the degradation and removal of nitrogen. Therefore, investigating the microbial community structures is quite necessary to understand the complex interactions during nitrogen removal and find ways to improve the design and operation of wastewater treatment processes. Many methods have been developed to investigate and characterize the microbes in activated sludge, including culture-dependent and culture-independent approaches. The culture-based 
methods were traditionally used to isolate the microbes in the active sludge and many key microbes were reported [6, 7]. However, it is not only time-consuming, but also difficult to obtain the microbial community effectively, since $80-90 \%$ of microorganisms are unculturable [8]. Culture-independent approaches such as PCR-denaturing gradient gel electrophoresis (PCR-DGGE), fluorescence in situ hybridization, and quantitative PCR (qPCR) have been widely used to profile the microbial community in recent decades [9-12]. Zhao et al. [6] used the PCR-DGGE technology to investigate the microbial community in aerobic granular sludge, and observed that Thauera was the most dominant microorganism and was crucial to $\mathrm{N}$ removal. Sauder et al. [13] employed a combination of PCRDGGE and qPCR methods to determine ammonia-oxidizing microbes, including ammonia-oxidizing bacteria (AOB) and archaea (AOA) in biofilm from a municipal WWTP, and the results showed that the ammonia gradient determined the relative abundance of AOA and AOB. Although these approaches greatly increased our understanding of the microbial community, they are limited owing to the PCR bias or low throughput [5].

Recently, high-throughput sequencing technologies such as 454 or Illumina sequencing have been developed to comprehensively identify the microbial diversity in different samples, including activated sludge, soil, ocean water, and food [14-17]. In addition, it was found that the municipal activated sludge from different plants shared some dominant and core genera, such as Zoogloea and Prosthecobacter [18]. However, a recent study indicated that different industrial WWTPs exhibited distinct community structures [19], and the microbes in tannery wastewater were not resolved adequately.

In this study, the microbial community of tannery wastewater involved in nitrification was investigated by using Illumina MiSeq sequencing and the results were further validated by GPCR analysis. This research may further elucidate the microbial community structure and aid in developing promising strategies and proper management technologies for tannery wastewater treatment.

\section{Materials and Methods}

\section{Sludge Sample Collection}

Activated sludge samples were separately collected from three full-scale WWTPs treating tannery wastewater of Ruixing Leather Industry Co. Ltd. (Sample AS-A, located in Jiaxing City, Zhejiang Province, China), Dazhong Leather Industry Co. Ltd. (Sample AS-B, located in Jiaxing City, Zhejiang Province, China), and Meidiyang
Leather Industry Co. Ltd. (Sample AS-C, located in Suqian City, Jiangsu Province, China). The detailed information of operational parameters for each plant is shown in Table S1. For analysis of the microbial community involved in nitrification, the sludge samples for each plant were collected in triplicate from the aerobic tanks. The collected samples were shaken well and placed in several sterile centrifuge tubes, and then centrifuged at 12,000 rpm for $10 \mathrm{~min}$ at $4^{\circ} \mathrm{C}$ and stored at $-80^{\circ} \mathrm{C}$ before use.

\section{DNA Extraction and PCR Amplification}

Genomic DNA was extracted by using the Mag-Bind Soil DNA Kit (Omega Biotek, USA), and the DNA concentration was determined using a spectrometer (Qubit 2.0; Invitrogen, USA). For the eubacterial community, the V3-V4 region of the 16S rRNA gene was amplified using the following primers: 341F: 5'CCCTACACGACGCTCTTCCGATCTN(barcode)CCTACGGGNG GCWGCAG-3' and 805R: 5'-GACTGGAGTTCCTTGGCACCCGAG AATTCCAGACTACHVGGGTATCTAATCC-3' [20]. The archaeal community was specially analyzed using nested PCR. The primers Arch340F (5'-CCCTAYGGGGYGCASCAG-3') and Arch1000R (5'-GGCCATGCACYWCYTCTC-3') [21] were used for the first PCR amplification, and then the PCR product was used as a template in the second PCR using the primers Arch349F (5'-CCC TACACGACGCTCTTCCGATCTN(barcode)GYGCASCAGKCG MGAAW-3') and Arch806R (5'-GACTGGAGTTCCTTGGCACCC GAGAATTCCAGGACTACVSGGGTATCTAAT-3'). Barcodes unique to each sample were incorporated in the forward primers, allowing the identification of each sample [22]. The PCRs were run on an i-Cycler (BioRad, USA) under the conditions described by Li et al. [20] and Gantner et al. [21]. After PCR amplification, the PCR products of three triplicates were mixed and purified with a DNA purification kit (Gel Purification Kits; Sangon, China), and the concentrations were determined using a spectrometer (Qubit 2.0). The PCR products were sent to Sangon Co., Ltd., in Shanghai for sequencing using the Illumina MiSeq sequencing system (Illumina, USA).

\section{High-Throughput Sequencing and Data Analysis}

After sequencing, the primers and spacers were trimmed. The pair-end reads were joined using the Flash program, and the lowquality sequences were then removed [23]. The resulting sequences were screened for chimeras, and the PCR chimeras were checked and filtered out by using UCHIME [24]. Operational taxonomic units (OTUs) were categorized using CD-HIT at a 97\% sequence similarity threshold, and the taxonomic assignment of OTUs was performed by the RDP classifier with $50 \%$ confidence [25]. Rarefaction curves were generated, and Shannon, Chao 1, and abundance-based coverage estimator (ACE) indexes were calculated using the Mothur program to compare the microbial diversity and richness between these sludge samples. Hierarchical clustering analysis was performed using CLUSTER and visualized using TREEVIEW. 


\section{Quantitative PCR Analysis}

To validate the abundance of the total bacteria $A O B$ and AOA, qPCR was conducted with the fluorescent SYBR-Green method. Genomic DNA was extracted using the Mag-Bind Soil DNA Kit (Omega Biotek) according to the manufacturer's instructions. The primers used for amplification were listed in Table S2. The PCR was performed in a $25 \mu \mathrm{l}$ volume containing $0.5 \mu \mathrm{l}$ of DNA template, $0.5 \mu \mathrm{l}$ of forward primer $(10 \mu \mathrm{M}), 0.5 \mu \mathrm{l}$ of reverse primer $(10 \mu \mathrm{M}), 0.5 \mu \mathrm{l}$ of dNTP $(10 \mu \mathrm{M}), 2.510 \mu \mathrm{l}$ of $10 \times$ Taq Buffer, $2 \mu \mathrm{l}$ of $\mathrm{MgCl}_{2}(25 \mu \mathrm{M}), 0.2 \mu \mathrm{l}$ of Taq-polymerase ( $5 \mathrm{U} / \mu \mathrm{l}$ ) (Sangon, China), and $18.3 \mu \mathrm{l}$ of nuclease-free water. The PCR products were checked by gel electrophoresis in $2 \%$ agarose, and then were purified using the DNA Fragment Recovery Kit (TaKaRa, China). The purified PCR products were connected to the cloning vector (pMD 18-T; TaKaRa, China). The plasmid in positive clones was extracted and purified using the MiniBEST Plasmid Purification Kit (TaKaRa, China), and the concentration was measured with a spectrophotometer (TU-1901; Puxi, China). Copy numbers were calculated on the basis of the mass concentration and average molecular weight of the plasmid. Ten-fold serial dilutions of known copy number were used as standard DNA. Quantitative PCR was performed in a total volume of $20 \mu \mathrm{l}$ reaction mixtures containing $10 \mu \mathrm{l}$ of SYBR Green qPCR Master Mix (Takara, China), $0.4 \mu \mathrm{l}$ of of each primer $(10 \mu \mathrm{M}), 2 \mu \mathrm{l}$ of template DNA, and $7.2 \mu \mathrm{l}$ of nuclease-free water. Each sample had three replicates, and the qPCR was performed by using a LightCycler 480 Real-time PCR System (Roche, Germany) with the following protocol: $95^{\circ} \mathrm{C}$ for $3 \mathrm{~min}$, followed by at $45 \mathrm{cycles}$ at $95^{\circ} \mathrm{C}$ for $15 \mathrm{sec}, 57^{\circ} \mathrm{C}$ for $20 \mathrm{sec}$, and $72^{\circ} \mathrm{C}$ for $30 \mathrm{sec}$.

\section{Statistical Analysis}

Statistical analysis was performed with SPSS 17.0 software (SPSS Inc., USA). Samples with $p$-values of $\leq 0.05$ were considered to be statistically different.

\section{Results and Discussion}

\section{Overall Analysis of Illumina MiSeq Sequencing}

Illumina high-throughput sequencing was used to investigate the microbial community diversity and structure of activated sludge from tannery plants. After removing low-quality sequences and chimeras, at least 25,714 (eubacteria) and 34,658 (archaea) effective sequences were obtained for each sample. The sequence number of each sample was normalized and tags with $97 \%$ similarity were then grouped into OTUs by the CD-HIT clustering method. At least 2,035 and 364 OTUs were generated from the eubacterial and archaeal sequences, respectively (Table 1). However, the rarefaction curves did not reach the plateau (Fig. S1), suggesting that there might be some microbes that had remained unidentified, and similar results were reported by Ma et al. [25] and Wang et al. [26]. Further analysis showed that a total of 457 and 31 OTUs for eubacteria and archaea, respectively, were shared by all plants (Fig. S2). Hierarchical clustering analysis indicated that the triplicate samples of each plant were clustered, which verified that the sequencing results were reproducible and reliable (Fig. 1).

In addition, the total number of OTUs was estimated by $\mathrm{ACE}$ and Chao 1 indexes, which represent the community richness. As for eubacteria, sample AS-A exhibited the highest ACE estimator and Chao 1 estimator, suggesting that AS-A has the greatest richness than AS-B and AS-C, whereas sample AS-C displayed the highest richness in archaea among all samples (Table 1). Microbial diversity was evaluated by Shannon index, and the higher the Shannon index, the richer the diversity [27]. In comparison, higher values of the Shannon index were achieved in samples AS-A (6.05) and AS-C (3.09) for the eubacteria and archaeal community, respectively. These results showed that samples AS-A and AS-C had higher eubacterial and archaeal diversities, respectively. In this study, relatively high Shannon indexes were obtained for all samples expect archaeal community in sample AS-B, corresponding to high OTU values. The diversity and richness of the tannery wastewater were similar to those of municipal wastewater plants and significantly higher than those of other industrial

Table 1. Summary of sequencing data for activated sludge samples collected from three tannery plants.

\begin{tabular}{|c|c|c|c|c|c|c|}
\hline & \multicolumn{2}{|c|}{ AS-A } & \multicolumn{2}{|c|}{ AS-B } & \multicolumn{2}{|c|}{ AS-C } \\
\hline & Eubacteria & Archaea & Eubacteria & Archaea & Eubacteria & Archaea \\
\hline Number of sequences & $27,997 \pm 1,738$ & $35,714 \pm 2,401$ & $25,759 \pm 2,144$ & $34,658 \pm 274$ & $27,791 \pm 970$ & $36,506 \pm 84$ \\
\hline Operational taxonomic units* & $3,016 \pm 145^{\mathrm{d}_{* *}}$ & $843 \pm 33^{b}$ & $2,035 \pm 81^{\mathrm{c}}$ & $364 \pm 14^{\mathrm{a}}$ & $2,335 \pm 49^{\mathrm{c}}$ & $1,004 \pm 265^{\mathrm{b}}$ \\
\hline Chao 1 richness estimator* & $8,903 \pm 1276^{\mathrm{a}}$ & $8,149 \pm 1,723^{\mathrm{ab}}$ & $6,918 \pm 371^{\mathrm{ab}}$ & $4,312 \pm 589^{b}$ & $7,067 \pm 322^{\mathrm{ab}}$ & $8,468 \pm 3,218^{\mathrm{ab}}$ \\
\hline ACE richness estimator* & $13,489 \pm 2,120^{\mathrm{ab}}$ & 22,703 & $10,287 \pm 1,355^{\mathrm{b}}$ & $19,338 \pm 4,108^{\mathrm{c}}$ & $10,246 \pm 161^{\mathrm{a}}$ & $29,059 \pm 2,265^{\mathrm{c}}$ \\
\hline
\end{tabular}

*They were defined by the $3 \%$ dissimilarity level (i.e., $97 \%$ identity threshold).

${ }^{* *}$ Different letters represent significant differences (one-way ANOVA, Turkey's test, $p<0.05$ ). 

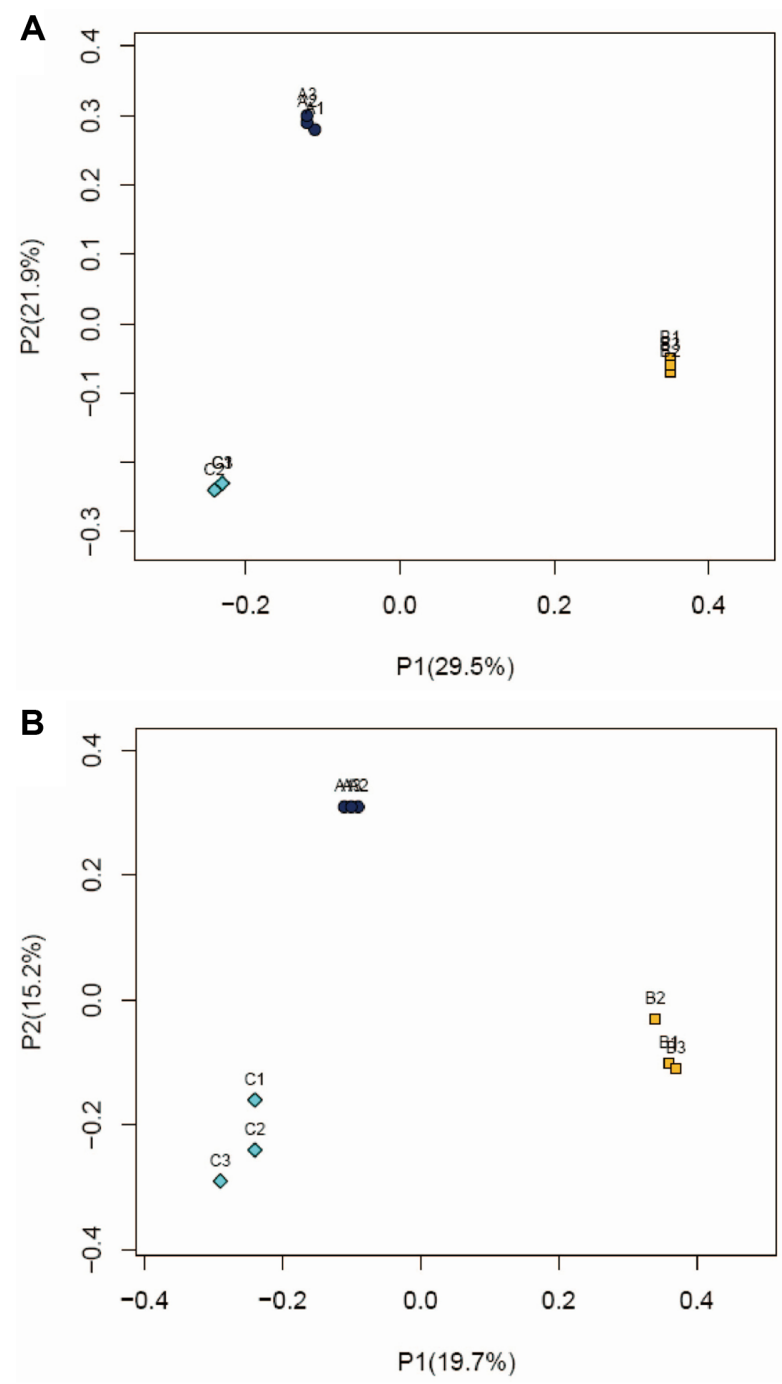

Fig. 1. Hierarchical clustering analysis of the activated sludge communities at the operational taxonomic unit level (triplicate samples for each plant).

A, B, and C in the figures represent samples AS-A, AS-B, and AS-C, respectively. (A) Eubacterial community. (B) Archaeal community.

wastewaters [15, 18, 25]. Ma et al. [25] investigated the bacterial community in coking wastewater, and the Shannon indexes were in the range of 3.0-4.0, corresponding to low OTU values ranging from 365 to 776 . Moreover, nearly all the sequences (over 99.99\%) were assigned to bacteria and only a few sequences belonged to archaea.

\section{Microbial Community Analysis at the Taxonomic Levels}

To identify the taxonomic diversity of the microbial community in activated sludge involved in nitrification from tannery wastewater, the RDP classifier was used to
A

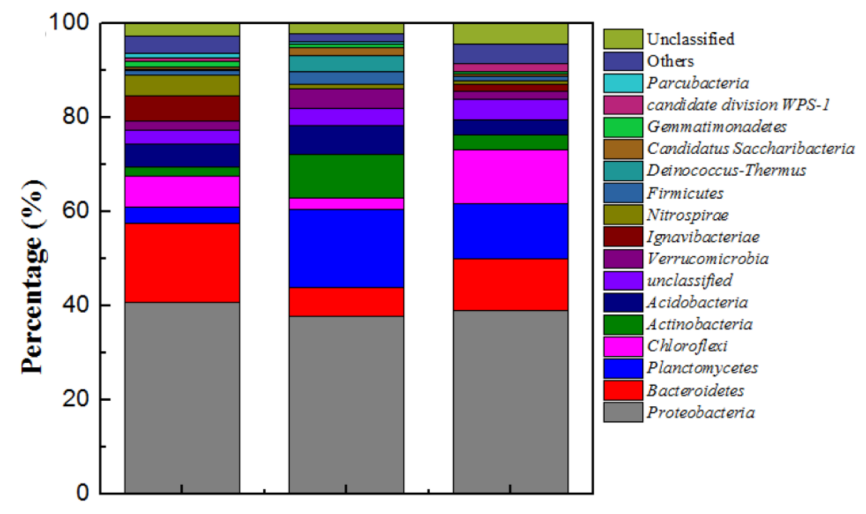

B

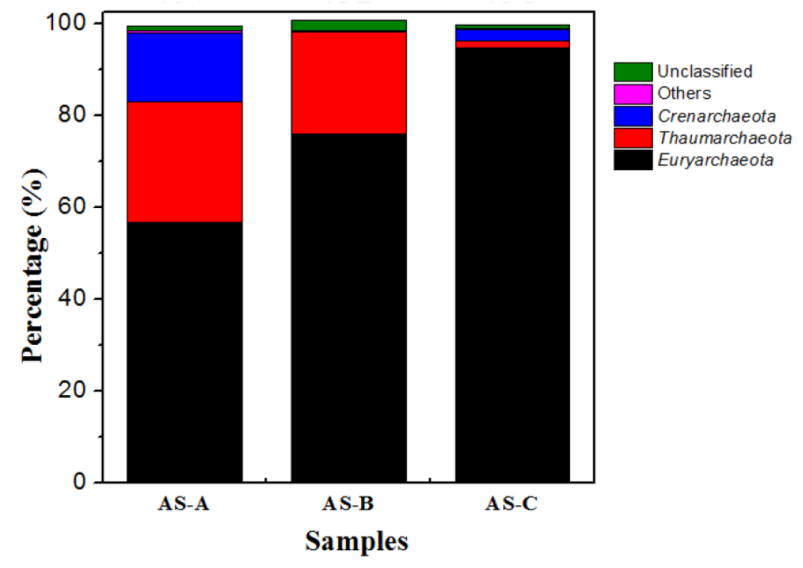

Fig. 2. Percentages of the predominant phylogenetic groups of activated sludge from tannery wastewater at the phylum level. Relative abundance was defined as the number of sequences assigned to that taxon divided by the total number of sequences per sample, and only the abundance higher than $1 \%$ in at least one sample was presented. (A) Eubacterial community. (B) Archaeal community.

assign the sequence tags to different taxonomic levels (from phylum to genus) at 50\% threshold. At the phylum level for the eubacterial community, 26 (AS-A), 23 (AS-B), and 24 (AS-C) bacterial phyla were detected and the phyla with relative abundance higher than $1 \%$ in at least one sample were provided (Fig. 2). As shown in Fig. 2A, Proteobacteria was the dominant phylum in all samples, accounting for $37-41 \%$ of total bacterial sequences, and this was consistent with the microbial composition in activated sludge treating tannery wastewater [28]. In addition, similar results were also reported in previous studies, which showed that Proteobacteria was the prominent phylum in soil, sediments, and activated sludge from municipal WWTPs and industrial WWTPs such as pharmaceutical, petroleum refinery, and coking wastewaters 
[25, 28-31]. Other major phyla were Bacteroidetes (6.04$16.80 \%)$, Planctomycetes (3.65-16.55\%), Chloroflexi (2.51$11.48 \%$ ), Actinobacteria (1.91-9.21\%), and Acidobacteria (3.04-6.20\%). These five phyla accounted for approximately $33.81-40.50 \%$ of the bacterial sequences within three samples, which were also present in other systems [25, 26, $28,29]$. Generally, members of these phyla were dominant in industrial WWTPs, which could be explained by their spore-forming ability, which could maintain survival during stressful environments [28, 32].

Although Proteobacteria (mostly Alpha-, Beta-, Gamma-, and Epsilon-proteobacteria) dominated in all samples, they had little in common in terms of constitute at the class level. Within Proteobacteria, Beta-proteobacteria was the most abundant class with the sequence percentages ranging from $9.66 \%$ to $17.44 \%$ (Fig. 3A). This finding was similar to the results of previous studies, which demonstrated that the Beta-subdivisions was the largest one within Proteobacteria in the activated sludge sample [25, 29].
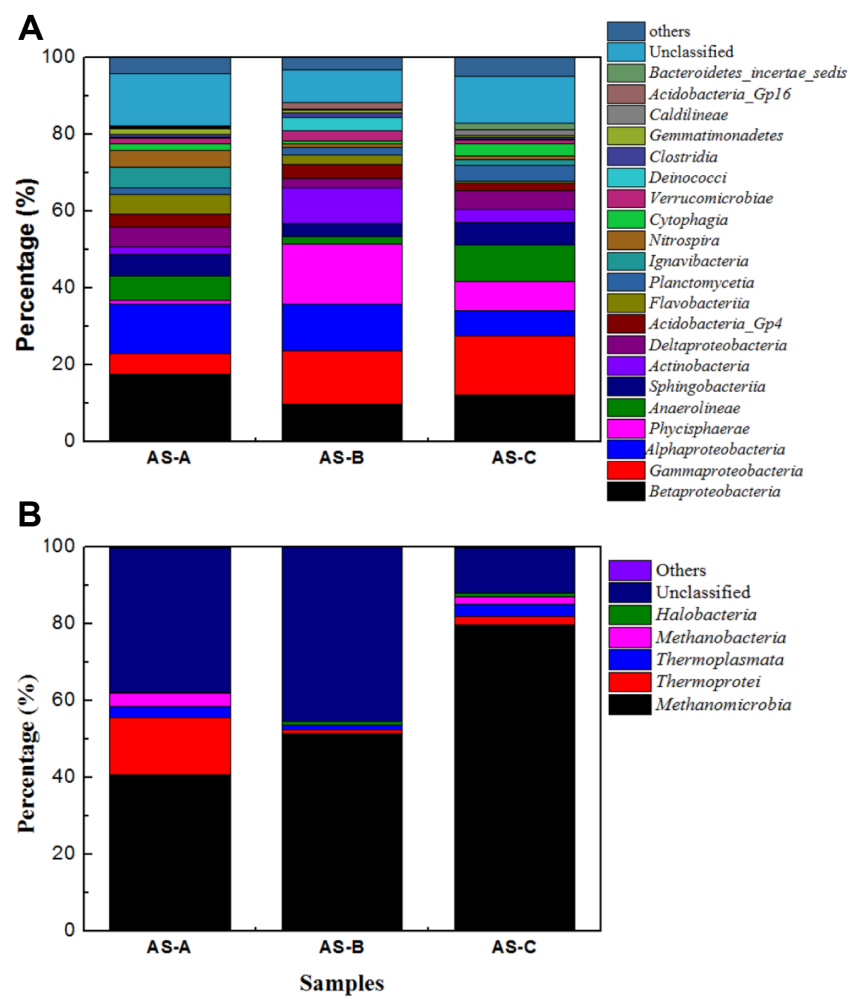

Fig. 3. Percentages of the predominant phylogenetic groups of activated sludge from tannery wastewater at the class level. Relative abundance was defined as the number of sequences assigned to that taxon divided by the total number of sequences per sample, and only the abundance higher than $1 \%$ in at least one sample was presented. (A) Eubacterial community. (B) Archaeal community.
Moreover, members of the Beta-proteobacteria were highly versatile in pollutant degradation and were detected in various biotreatment systems [25, 33]. Alpha-proteobacteria and Gamma-proteobacteria were also reported as the major phyla elsewhere, and the percentages were 6.68-13.06\% and $5.34-15.32 \%$, respectively. Other research reported different observations, where Alpha-proteobacteria was the main subdivision of Proteobacteria samples from wastewater treatment systems [28]. This disparity may be due to the difference in wastewater characteristics and treatment processes. Except for the subdivisions of Proteobacteria, other main classes were Phycisphaerae (1.05-15.63), Anaerolineae (2.0-9.44\%), Sphingobacteria (3.52-6.0\%), and Actinobacteria (1.86-9.08\%).

At the family level, a total of 234 families were identified, and the majority of sequences belonged to 21 families ( $>1 \%$ on average) (Fig. 4). As shown in Fig. 4A, a large variation of relative community abundance among three samples was observed, suggesting a significant influence of physiological characterization on microbial community structures at the
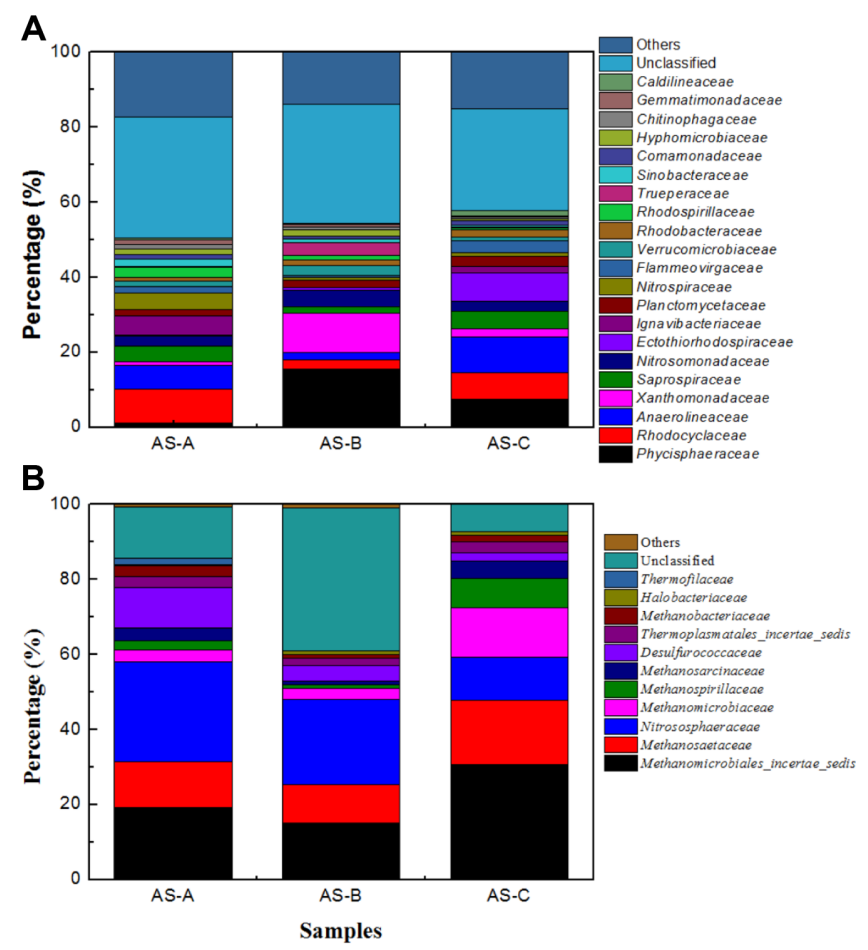

Fig. 4. Percentages of the predominant phylogenetic groups of activated sludge from tannery wastewater at the family level. Relative abundance was defined as the number of sequences assigned to that taxon divided by the total number of sequences per sample, and only the abundance higher than $1 \%$ in at least one sample was presented. (A) Eubacterial community. (B) Archaeal community. 
family level. As for AS-A, Rhodocyclaceae, Anaerolineaceae, and Ignavibacteriaceae were the main families, which accounted for $9.18 \%, 6.25 \%$, and $5.17 \%$ of the total sequences, respectively. Phycisphaeraceae (15.63\%), Xanthomonadaceae $(10.58 \%)$, and Nitrosomonadaceae $(4.46 \%)$ predominated in sample AS-B. The dominant families in AS-C were Anaerolineaceae (9.44\%), Ectothiorhodospiraceae (7.74\%), Phycisphaeraceae (7.56\%), and Rhodocyclaceae (6.99\%). Among these families, Rhodocyclaceae and Nitrosomonadaceae belong to Beta-proteobacteria, whereas Xanthomonadaceae and Ectothiorhodospiraceae belong to Gamma-proteobacteria. Rhodocyclaceae was a core family in many WWTPs, which was reported to be responsible for the denitrifying and aromatic-degrading process $[25,29,34]$.

As for the archaea, the most abundant phyla were Euryarchaeota (56.87-94.92\%), Thaumarchaeota (1.47$26.28 \%$ ), and Crenarchaeota (0.06-14.99\%), which accounted for approximately $98 \%$ of all the archaeal sequences (Fig. 2B). Among them, Euryarchaeota and Thaumarchaeota were also detected previously as the predominant phyla in tannery WWTPs by a combined PCR-DGGE and highthroughput sequencing method [28]. At the class level, the dominant class was Methanomicrobia with the abundance ranging from $40.7 \%$ to $89.68 \%$ (Fig. 3B). The largest family in AS-A and AS-B were Nitrososphaeraceae, whereas in sample AS-C, Methanomicrobiale sincertae-sedis was the abundant family (Fig. 4B). Although a relatively low abundance of archaea was detected, they may play important roles during nitrification. In addition, it was worth noting the potential correlation between the bacteria and archaea in the wastewater treatment process.

\section{Identification of the Core Genera}

Only 54 eubacterial genera and 14 archaeal genera were shared by all samples, and 35 and 14 most abundant eubacterial and archaeal genera were selected to assess the microbial community distribution, respectively (Table S2, Fig. 5). As shown in Fig. 5, there was a distinct difference in the microbial composition in each sample. Thauera, Gp4, Ignavibacterium, and Nitrospira were dominant genera in sample AS-A, which accounted for $4.74 \%, 3.38 \%, 4.57 \%$, and $4.44 \%$ of the total identified eubacterial sequences, respectively. As for sample AS-B, Phycisphaera, Nitrosomonas, Gp4, and Truepera were the main genera, with $15.63 \%$, $3.94 \%, 3.38 \%$, and $3.46 \%$ of the sequences on average. In sample AS-C, Phycisphaera (8.34\%), Gp4 (3.52\%), Arenimonas $(3.41 \%)$, and Thauera $(3.08 \%)$ were the predominant genera.

The genus Thauera, of the class Beta-proteobacteria, was the primary genus in AS-A, and it was detected in all the
A

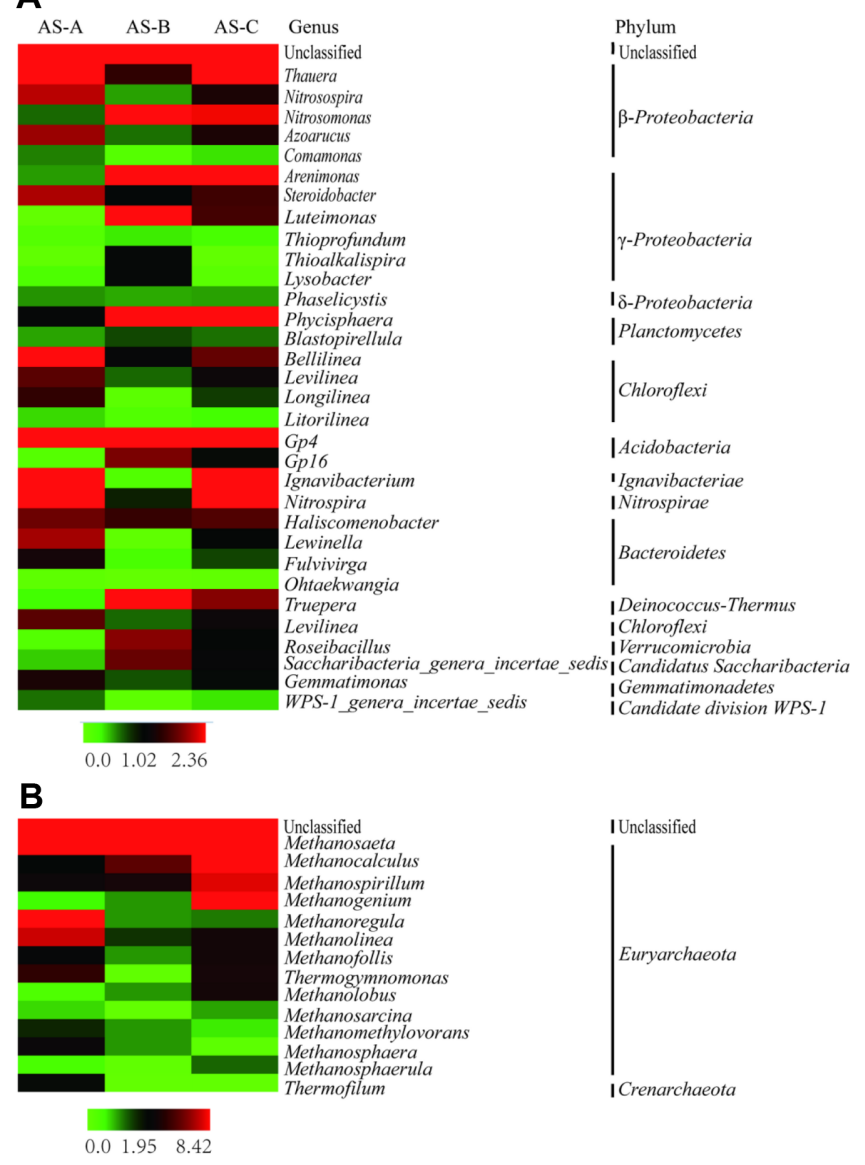

Fig. 5. Heat map of the main genera in activated sludge (with relative abundance over $1 \%$ in at least one sample).

The color intensity in each panel indicates the relative abundance of a genus in each sample, and the abundance was defined as the number of sequences assigned to that taxon divided by the total number of sequences per sample.

three samples (Fig. 5A). Similar results were also reported in aerobic sludge samples collected from tannery WWTPs, and extremely high abundance was detected $(4.64 \%-$ $15.81 \%$ ) [26]. In addition, Thauera bacteria were frequently detected in other wastewater treatment bioreactors, and they were considered to be functionally important populations [15, 32]. In a recent study, the genus Thauera was identified as the most abundant microorganism in a granular sludge system, and it was proved to harbor perfect nitrification ability, with a $99.8 \% \mathrm{NH}_{4}{ }^{+} \mathrm{N}(50 \mathrm{mg} / \mathrm{l})$ removal efficiency in $48 \mathrm{~h}$ [6]. With the exception of nitrification ability, Thauera was also known to be capable of denitrification and organic compound biodegradation [15, 26]. Mao et al. [35] isolated three Thauera strains from a coking wastewater treatment system that could degrade 
phenol, methylphenol, and indole. The potential nitrification and denitrification abilities of these bacteria may contribute to the explanation for ammonia removal in tannery WWTPs.

Gp4, which belongs to phylum Acidobacteria, was another core genus detected in this study. As previously reported, Acidobacteria, particularly subgroups Gp1-Gp4 and Gp6, represented about $20 \%$ of the bacteria in forest soil, and they were frequently detected in activated sludge of industrial and municipal WWTPs [25, 36]. Wang et al. [30] investigated the bacterial diversity in 14 wastewater treatment systems by pyrosequencing, and Gp4 and Gp6 were the core genera shared by 12 wastewater treatment systems. In the present research, Gp4 was also observed as the major constituent in all samples $(3.38 \%, 3.65 \%$, and $3.52 \%$ ). Unfortunately, the genus Gp4 is not well described, and knowledge concerning the roles of Gp4 in biological wastewater treatment systems is limited.

Phycisphaera was the largest genus in both AS-B and ASC (15.65\% and $8.34 \%)$, and it exhibited high abundance in a membrane bioreactor for organic matter removal [37]. In addition, the genus Phycisphaera was the main portion in an autotrophic nitrogen bioreactor [38]. Genus Ignavibacteria exhibited relatively high percentage in samples AS-A and AS-C, and it is a group of chemoheterotrophs with a versatile metabolism [39]. In a full-scale anaerobic/anoxic/ oxic (A2O) nitrogen and phosphorus removal reactor, Ignavibacteria ranked the fourth abundant genus $(10,883$ reads, $4.09 \%$ ) [40].

For the archaea, the largest genus was Methanosaeta $(10.08-27.23 \%)$ in all the three samples, which was affiliated to phylum Euryarchaeota. Previous research demonstrated that genus Methanosaeta generally existed in the anaerobic digestion of organic compounds, converting acetate to methane [41], and further research is needed to elucidate the role of genus Methanosaeta during nitrification.

\section{Abundance of AOA and $\mathrm{AOB}$}

As is known, nitrification is an aerobic process that oxidizes $\mathrm{NH}_{4}^{+}$to $\mathrm{NO}_{2}{ }^{-}$with the help of $\mathrm{AOB}$, followed by the conversion of nitrite to nitrate by nitrite-oxidizing bacteria (NOB) [42]. Both $\mathrm{AOB}$ and NOB are the two important types of nitrifiers, and were frequently detected in activated sludge $[4,5,25]$. Nitrosospira and Nitrosomonas were the most important genera of $\mathrm{AOB}$, which are postulated as the main contributors to ammonia oxidation [25]. As expected, all the samples exhibited a relatively high abundance of Nitrosospira and Nitrosomonas (Fig. 3, and Table S2). Fig. 6 shows the sequence numbers of Nitrosospira and Nitrosomonas in the three samples. The

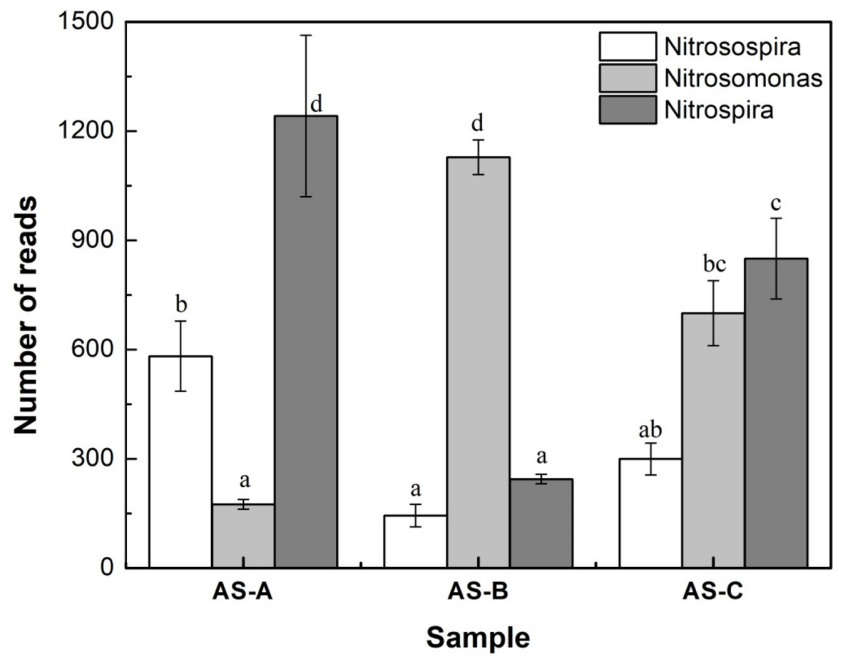

Fig. 6. Sequence numbers of Nitrosomonas, Nitrosospira, and Nitrospira in each sample revealed by the metagenomic approach.

Different letters represent significant differences (one-way ANOVA, Turkey's test, $p<0.05)$.

highest number of Nitrosospira was detected in AS-A, and sample AS-B had the most of Nitrosomonas. A detailed analysis of the number of $\mathrm{AOB}$ indicated that the number of Nitrosomonas was higher than that of Nitrosospira in all samples except in AS-A. Previous studies have revealed that AOB Nitrosomonas instead of Nitrosospira dominates in WWTPs owing to the high resistance to the changing environments and high growth rate compared with Nitrosospira [26, 43].

NOB included the four groups Nitrobacter, Nitrospina, Nitrococcus, and Nitrospira, belonging to Alpha-proteobacteria, Gamma-proteobacteria, Beta-proteobacteria, and Nitrospirae, respectively [44]. In this study, only Nitrospira was detected in the three samples, with high distribution in AS-A and AS-C, suggesting that Nitrospira is the major NOB in tannery WWTPs. This result was in agreement with that obtained in other activated sludge $[25,44]$.

Members of the AOA are another kind of nitrifiers, catalyzing the conversion of $\mathrm{NH}_{4}^{+}$to nitrite. Unfortunately, no AOA was identified by sequencing analysis. The same failure was also encountered in other bioreactors [25]. In this study, the number of AOB exceeded that of AOA in activated sludge, and this may be ascribed to the high ammonia concentration and high ammonia loading in tannery WWTPs. Usually, AOA are more abundant than AOB in environments with low ammonia concentrations, such as soil, seawater, and sediments $[45,46]$. Based on 


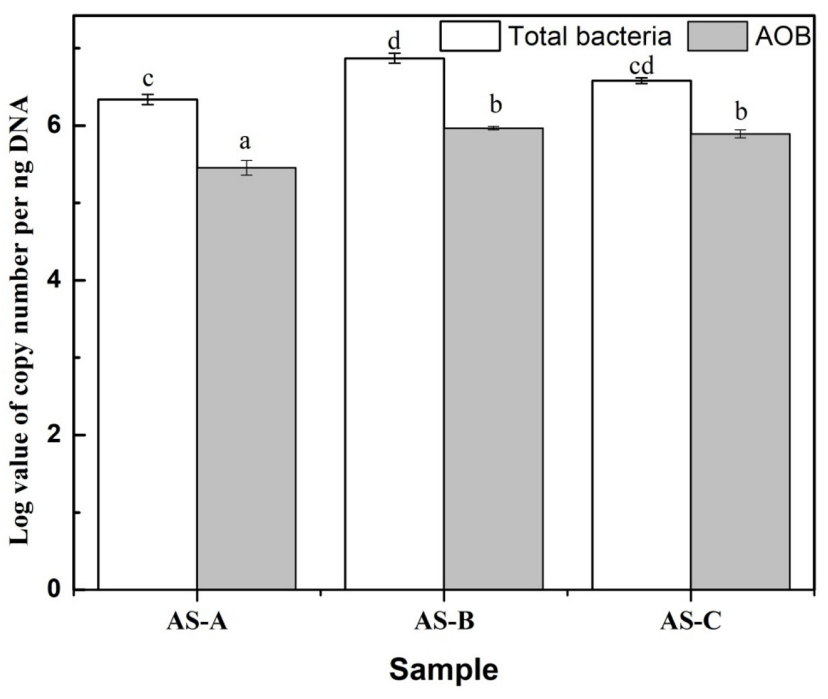

Fig. 7. Abundance of $a m o \mathrm{~A}$ and $16 \mathrm{~S}$ rRNA in the three samples as revealed by qPCR.

Error bars represent the standard deviation calculated from three independent assays. Different letters represented significant differences (one-way ANOVA, Turkey's test, $p<0.05$ ).

these results and previous reports, it could be inferred that AOB may be more competitive than AOA in activated sludge systems and function as the main contributors for nitrification in tannery WWTPs.

To validate the sequencing result, $\mathrm{qPCR}$ assay was conducted to quantify the abundance of $\mathrm{AOA}, \mathrm{AOB}$, and total bacteria in the three samples (Fig. 7). As shown in Fig. 7, the quantification of AOA amoA in all samples was not successful, and this result was consistent with that of the metagenomic sequencing. The fact may probably be due to the low abundance and PCR bias in activated sludge. In addition, previous studies also revealed that AOA amoA is absent in aerobic sludge of different WWTPs and it was detected only in the anaerobic sludge [26, 47]. Analysis of the abundance of $\mathrm{AOB}$ and total bacteria showed that the log copy numbers of the $\mathrm{AOB}$ amoA gene and bacterial $16 \mathrm{~S}$ rRNA in the three samples were 5.4-6.0 and 6.3-6.6 copies per nanogram of DNA, respectively (Fig. 7). It was shown that the qPCR quantification results were in agreement with the sequencing ones.

In this study, the microbial community of activated sludge involved in nitrification from tannery WWTPs was investigated by the Illumina MiSeq sequencing approach. The results showed that the diversity and richness of the tannery wastewater were similar to those of municipal wastewater and significantly higher than those of other industrial wastewaters. The taxonomic diversity of the microbial community of tannery wastewater was identified from the phylum to genus levels, and the core genera were further discussed in the present manuscript. qPCR was performed to quantify the abundance of the amo $A$ gene of $\mathrm{AOB}$ and total bacteria, and the results were in agreement with the sequencing ones. The results obtained in this study can extend our knowledge about nitrifiers in tannery WWTPs, and might be practically useful in efficiently removing nitrogen from industrial wastewaters.

\section{Acknowledgments}

This study was funded by the Special Foundation for State Key Research and Development Program of China (2017YFB0308500), and by the National Natural Science Foundation of China (31671849).

\section{Conflict of Interest}

The authors have no financial conflicts of interest to declare.

\section{References}

1. Wang YN, Zeng Y, Zhou J, Zhang W, Liao X, Shi B. 2016. An integrated cleaner beamhouse process for minimization of nitrogen pollution in leather manufacture. J. Clean Prod. 112: 2-8.

2. Wang Y, Zeng Y, Chai X, Liao X, He Q. 2012. Ammonia nitrogen in tannery wastewater: distribution, origin, and prevention. J. Am. Leather Chem. Assoc. 107: 40-50.

3. Zhou J, Wang YN, Zhang W, Bi S. 2014. Nutrient balance in aerobic biological treatment of tannery wastewater. J. Am. Leather Chem. Assoc. 109: 154-160.

4. Peng X, Guo F, Ju F, Zhang T. 2014. Shifts in the microbial community, nitrifiers and denitrifiers in the biofilm in a full-scale rotating biological contactor. Environ. Sci. Technol. 48: 8044-8052.

5. Ye L, Zhang T, Wang T, Fang Z. 2012. Microbial structures, functions, and metabolic pathways in wastewater treatment bioreactors revealed using high-throughput sequencing. Environ. Sci. Technol. 46: 13244-13252.

6. Zhao Y, Huang J, Hai Z, Hua Y. 2013. Microbial community and $\mathrm{N}$ removal of aerobic granular sludge at high COD and $\mathrm{N}$ loading rates. Bioresour. Technol. 143: 439-446.

7. Zhang S, Sha C, Jiang W, Li W, Zhang D, Li J, et al. 2015. Ammonium removal at low temperature by a newly isolated heterotrophic nitrifying and aerobic denitrifying bacterium Pseudomonas fluorescens wsw-1001. Environ. Technol. 36: 2488-2494. 
8. Amann RI, Ludwig W, Schleifer KH. 1995. Phylogenetic identification and in situ detection of individual microbial cells without cultivation. Microbiol. Rev. 59: 143-169.

9. Wang L, Liu J, Zhao Q, Wei W, Sun Y. 2016. Comparative study of wastewater treatment and nutrient recycle via activated sludge, microalgae and combination systems. Bioresour. Technol. 211: 1-5.

10. Waheed H, Hashmi I, Naveed AK, Khan SJ. 2013. Molecular detection of microbial community in a nitrifying-denitrifying activated sludge system. Int. Biodeterior. Biodegradation 85: 527-532.

11. Hu B, Zheng P, Tang C, Chen J, van der Biezen E, Zhang L, et al. 2010. Identification and quantification of anammox bacteria in eight nitrogen removal reactors. Water Res. 44: 5014-5020.

12. Ge S, Wang S, Xiong Y, Shuang Q, Li B, Peng Y. 2015. Detection of nitrifiers and evaluation of partial nitrification for wastewater treatment: a review. Chemosphere 140: 85-98.

13. Sauder LA, Peterse F, Schouten S, Neufeld JD. 2012. Lowammonia niche of ammonia-oxidizing archaea in rotating biological contactors of a municipal wastewater treatment plant. Environ. Microbiol. 14: 2589-2600.

14. Zheng X, Su Y, Li X, Xiao N, Wang D, Chen Y. 2013. Pyrosequencing reveals the key microorganisms involved in sludge alkaline fermentation for efficient short-chain fatty acids production. Environ. Sci. Technol. 47: 4262-4268.

15. Wang B, Peng Y, Guo Y, Zhao M, Wang S. 2016. Illumina MiSeq sequencing reveals the key microorganisms involved in partial nitritation followed by simultaneous sludge fermentation, denitrification and anammox process. Bioresour. Technol. 207: 118-125.

16. Tao Y, Li J, Rui J, Xu Z, Zhou Y, Hu X, et al. 2014. Prokaryotic communities in pit mud from different-aged cellars used for the production of Chinese strong-flavored liquor. Appl. Environ. Microbiol. 80: 2254-2260.

17. Zafra G, Taylor TD, Absalón AE, Cortés-Espinosa DV. 2016. Comparative metagenomic analysis of $\mathrm{PAH}$ degradation in soil by a mixed microbial consortium. J. Hazard. Mater. 318: $702-710$.

18. Zhang T, Shao MF, Ye L. 2012. 454 Pyrosequencing reveals bacterial diversity of activated sludge from 14 sewage treatment plants. ISME J. 6: 1137-1147.

19. Ibarbalz FM, Figuerola ELM, Erijman L. 2013. Industrial activated sludge exhibit unique bacterial community composition at high taxonomic ranks. Water Res. 47: 38543864.

20. Li D, Chen B, Zhang L, Gaur U, Ma T, Jie H, et al. 2016. The musk chemical composition and microbiota of Chinese forest musk deer males. Sci. Rep. 6: 18975.

21. Gantner S, Andersson AF, Alonso-Sáez L, Bertilsson S. 2011. Novel primers for $16 \mathrm{~S}$ rRNA-based archaeal community analyses in environmental samples. J. Microbiol. Methods 84: 12-18.
22. Zhang J, Lv C, Tong J, Liu J, Liu J, Yu D, et al. 2015. Optimization and microbial community analysis of anaerobic co-digestion of food waste and sewage sludge based on microwave pretreatment. Bioresour. Technol. 200: 253-261.

23. Magoč T, Salzberg SL. 2011. FLASH: fast length adjustment of short reads to improve genome assemblies. Bioinformatics 27: 2957-2963.

24. Edgar RC, Haas BJ, Clemente JC, Quince C, Knight R. 2011. UCHIME improves sensitivity and speed of chimera detection. Bioinformatics 27: 2194-2200.

25. Ma Q, Qu Y, Shen W, Zhang Z, Wang J, Liu Z, et al. 2015. Bacterial community compositions of coking wastewater treatment plants in steel industry revealed by Illumina highthroughput sequencing. Bioresour. Technol. 179: 436-443.

26. Wang Z, Zhang XX, Lu X, Liu B, Li Y, Long C, et al. 2014. Abundance and diversity of bacterial nitrifiers and denitrifiers and their functional genes in tannery wastewater treatment plants revealed by high-throughput sequencing. PLoS One 9: e113603.

27. Yang Q, Xiong P, Ding P, Chu L, Wang J. 2015. Treatment of petrochemical wastewater by microaerobic hydrolysis and anoxic/oxic processes and analysis of bacterial diversity. Bioresour. Technol. 196: 169-175.

28. Liang H, Ye D, Li P, Su T, Wu J, Luo L. 2016. Evolution of bacterial consortia in an integrated tannery wastewater treatment process. RSC Adv. 6: 87380-87388.

29. Zhang X, Qu Y, Ma Q, Zhang Z, Li D, Wang J, et al. 2015. Illumina MiSeq sequencing reveals diverse microbial communities of activated sludge systems stimulated by different aromatics for indigo biosynthesis from indole. PLoS One 10: $\mathrm{e} 0125732$.

30. Wang X, Hu M, Xia Y, Wen X, Ding K. 2012. Pyrosequencing analysis of bacterial diversity in 14 wastewater treatment systems in China. Appl. Environ. Microbiol. 78: 7042-7047.

31. Ibarbalz FM, Figuerola EL, Erijman L. 2013. Industrial activated sludge exhibit unique bacterial community composition at high taxonomic ranks. Water Res. 47: 38543864.

32. Shu D, He Y, Yue H, Wang Q. 2015. Microbial structures and community functions of anaerobic sludge in six fullscale wastewater treatment plants as revealed by 454 highthroughput pyrosequencing. Bioresour. Technol. 186: 163-172.

33. Desta AF, Assefa F, Leta S, Stomeo F, Wamalwa M, Njahira M, et al. 2015. Microbial community structure and diversity in an integrated system of anaerobic-aerobic reactors and a constructed wetland for the treatment of tannery wastewater in Modjo, Ethiopia. PLoS One 10: 0128053.

34. Loy A, Schulz C, Lücker S, Schöpfer-Wendels A, Stoecker K, Baranyi C, et al. 2005. 16S rRNA gene-based oligonucleotide microarray for environmental monitoring of the betaproteobacterial order "Rhodocyclales". Appl. Environ. Microbiol. 71: 1373-1386.

35. Mao Y, Zhang X, Xia X, Zhong H, Zhao L. 2010. Versatile 
aromatic compound-degrading capacity and microdiversity of Thauera strains isolated from a coking wastewater treatment bioreactor. J. Ind. Microbiol. Biotechnol. 37: 927-934.

36. Naether A, Foesel BU, Naegele V, Wüst PK, Weinert J, Bonkowski M, et al. 2012. Environmental factors affect acidobacterial communities below the subgroup level in grassland and forest soils. Appl. Environ. Microbiol. 78: 73987406 .

37. Zhuang H, Hong X, Han H, Shan S. 2016. Effect of pure oxygen fine bubbles on the organic matter removal and bacterial community evolution treating coal gasification wastewater by membrane bioreactor. Bioresour. Technol. 221: 262-269.

38. Gonzalez-Martinez A, Rodriguez-Sanchez A, Rodelas B, Abbas BA, Martinez-Toledo MV, Van Loosdrecht $\mathrm{M}$, et al. 2015. 454-Pyrosequencing analysis of bacterial communities from autotrophic nitrogen removal bioreactors utilizing universal primers: effect of annealing temperature. BioMed. Res. Int. 2015: 1-12.

39. Liu Z, Frigaard N-U, Vogl K, lino T, Ohkuma M, Overmann J, et al. 2012. Complete genome of Ignavibacterium album, a metabolically versatile, flagellated, facultative anaerobe from the phylum Chlorobi. Front. Microbiol. 3: 1-15.

40. Tian M, Zhao F, Shen X, Chu K, Wang J, Chen S, et al. 2015. The first metagenome of activated sludge from full-scale anaerobic/anoxic/oxic (A2O) nitrogen and phosphorus removal reactor using Illumina sequencing. J. Environ. Sci.
35: 181-190.

41. Kamagata Y, Mikami E. 1991. Isolation and characterization of a novel thermophilic Methanosaeta strain. Int. J. Syst. Evol. Microbiol. 41: 191-196.

42. Siripong S, Rittmann BE. 2007. Diversity study of nitrifying bacteria in full-scale municipal wastewater treatment plants. Water Res. 41: 1110-1120.

43. Bai Y, Sun Q, Wen D, Tang X. 2012. Abundance of ammonia-oxidizing bacteria and archaea in industrial and domestic wastewater treatment systems. FEMS Microbiol. Ecol. 80: 323-330.

44. Duan L, Song Y, Xia S, Hermanowicz SW. 2013. Characterization of nitrifying microbial community in a submerged membrane bioreactor at short solids retention times. Bioresour. Technol. 149: 200-207.

45. Martens-Habbena W, Berube PM, Urakawa H, de La Torre JR, Stahl DA. 2009. Ammonia oxidation kinetics determine niche separation of nitrifying Archaea and Bacteria. Nature 461: 976-979.

46. Wuchter C, Abbas B, Coolen MJ, Herfort L, van Bleijswijk J, Timmers $\mathrm{P}$, et al. 2006. Archaeal nitrification in the ocean. Proc. Natl. Acad. Sci. USA 103: 12317-12322.

47. Zhang T, Ye L, Tong AHY, Shao M-F, Lok S. 2011. Ammoniaoxidizing archaea and ammonia-oxidizing bacteria in six full-scale wastewater treatment bioreactors. Appl. Microbiol. Biotechnol. 91: 1215-1225. 